\title{
Cardiac troponin T3: A distinguishing parameter between contractile and conducting cardiac fibers in adult male mice
}

\author{
Original \\ Article \\ Mohamed E. Ali Khalifa ${ }^{1}$ and Nesma I. El-Naseery ${ }^{2}$ \\ ${ }^{1}$ Department of Histology and Cell Biology, Faculty of Medicine, ${ }^{2}$ Department of Histology \\ and Cytology, Faculty of Veterinary Medicine Zagazig University, Zagazig, Egypt
}

\begin{abstract}
Introduction: Cardiac diseases can affect the contractile and/or conductive cardiac muscle cells. Recently, the microscopic examination of the subendocardial biopsy is being used in the diagnosis of some cardiac lesions. However, the routine staining cannot differentiate between different types of cardiac fibers.

Aim of the work: The present study aims to illustrate the use of anti-cardiac troponin-T3 (cTnT3) immunostaining as a recent tool to distinguish between the contractile and conducting fibers.

Materials and Methods: Specimens from both left ventricular wall and interventricular septum were obtained from 6 healthy adult male mice. These specimens were fixed in $4 \%$ paraformaldehyde at $4^{\circ} \mathrm{C}$ and processed for paraffin sections. Then, 3 $\mu \mathrm{m}$ thick sections were stained with H\&E and anti-cTnT3 antibody and were examined under light microscope. The optical density of the cTnT3 staining affinity of the cardiac fibers was measured morphometrically and the values were statistically analyzed.

Results: Examination of H\&E stained sections of the left ventricular wall and interventricular septum revealed homogenous staining of all cardiac fibers with an undetectable difference among them. While examination of the immunostained sections with anti-cTnT3 revealed different affinities: the majority of fibers expressed high- positive staining affinity while other fibers had a low or no staining affinity. The high- positive staining affinity was in the form of cytoplasmic transverse dark brownish striations in longitudinally oriented cardiac fibers. The fibers which expressed a low or no staining affinity were either singly scattered especially in the left ventricular wall or grouped in the subendocardial regions of the interventricular septum. Statistically, the optical density values of a low or no staining affinity cardiac fibers were significantly lower (2.5 \pm 0.01$)$ than high positive staining affinity cardiac fibers (3.04 \pm 0.19$)$.

Conclusion: Immunostaining with anti-cTnT3 staining could be added to the routine staining in the examination of cardiac biopsies.
\end{abstract}

Received: 08 August 2017, Accepted: 24 February 2018

Key Words: Contractile fibers, conducting fibers, mice, troponin-T3.

Corresponding Author: Mohamed E. Ali Khalifa, Department of Histology and Cell Biology, Faculty of Medicine, Zagazig University, Zagazig, Egypt, Tel.: 00201064289893, E-mail: Khalkhalifa@yahoo.com.

ISSN: 1110-0559, Vol. 41, No.1

\section{INTRODUCTION}

Myocardial disorders, which have unique prognoses and treatment, are seldom diagnosed by noninvasive testing. Therefore, a cardiac biopsy is urgently needed in the diagnosis of adult and pediatric cardiovascular diseases. The microscopic examination of the myocardium may yield clinically useful information in many disorders as cardiomyopathy, myocarditis, and rejection after heart transplantation $^{[1-3]}$.

The function of a contractile tissue is directly related to contractile fibers ${ }^{[4]}$. But, the function of a healthy heart depends on contractile, conducting and endocrinal fibers. The contractile fibers (CF) are responsible for the pumping action $^{[5]}$. However, the cardiac conduction system (CCS) is responsible for the initiation, coordination, and conduction of the heartbeat ${ }^{[6]}$. Many researchers have paid considerable attention to discriminative techniques between $\mathrm{CF}$ and
CCS that can be used to describe their normal function and predict dysfunction with pathology or malformation ${ }^{[7]}$. The traditional stain methods of gross dissection are Periodic Acid-Schiff (PAS) ${ }^{[8]}$ and immunofluorescence staining ${ }^{[9]}$. In addition, the micro-computed tomography (microCT)" a modality to resolve soft tissue internal structure" resolves the 3-dimensional morphology of CCS but its ability to discriminate between different cardiac tissues is limited compared to traditional staining ${ }^{[10]}$. Our present understanding of cardiac histology and functions owes much to previous investigations ${ }^{[11,12]}$.

Cardiac troponin $\mathrm{T}(\mathrm{TnT})$, a part of the troponin complex (TI, TC, TT) in skeletal and cardiac muscle fibers, binds to tropomyosin, interlocking them to form a troponin-tropomyosin complex. While, the rest of the complex modulates muscular contraction ${ }^{[13]}$. Four human cardiac TnT (cTnT) isoforms were determined. Of them, TnT1 is the mainly expressed in fetal heart, while TnT3 
dominants in the adult heart ${ }^{[14]}$. Besides, TnT4 exists in the failing adult heart and cTnT releases rapidly from injured cardiac muscles. Noteworthy, presence of measurable amounts of circulating cTnT can indicate cardiac damage. Serologically, cTnT is a proven diagnostic and risk biomarker in acute coronary syndromes ${ }^{[15]}$. On the other hand, histologically, cTnT is sensitive to detect acute myocardial infarction as the infracted fibers show a low staining affinity ${ }^{[16]}$.

Depending on TnT3-low staining affinity of conducting myofibers in contrast to contractile fibers which have high staining affinity ${ }^{[17]}$, the present study was designed to differentiate between the contractile and conducing myofibers in the adult mouse heart by immunohistochemical staining of cTnT3.

\section{MATERIALS AND METHODS}

\section{Animals}

In the present study, 6 healthy 12 -weeks old male mice (70-85g) were purchased from animal house of Faculty of Medicine, Zagazig University, Egypt. These animals were housed in a controlled environment (room temperature of $22 \pm 4 \mathrm{C}$, a relative humidity of $55 \pm 20 \%$, and a 12 -h light/12-h dark cycle). The mice had free access to tap water and an adequate rodent diet. In handling the experimental animals, we adhered to the Guide for the Care and Use of Laboratory Animals, Zagazig University, Egypt.

\section{Histological analysis}

Specimens were prepared for microscopic examinations ${ }^{[18]}$. In brief, after deep inhalation anesthesia, the hearts were removed and cut transversally into atria and ventricles. Then, the ventricles were fixed in $4 \%$ paraformaldehyde at $4^{\circ} \mathrm{C}$ for $4 \mathrm{~h}$. The specimens were dehydrated in an ascending graded ethanol, cleared in xylene and embedded in paraffin wax. Subsequently, the obtained $3 \mu \mathrm{m}$ thick paraffin sections were deparaffinized, rehydrated, and stained with hematoxylin and eosin (H\&E).

\section{Immunohistochemical analysis}

To examine the cardiac affinity to anti-cTnT3, a mouse monoclonal anti-cTnT3 antibody (ab-10214, Abcam, Tokyo, Japan) was used ${ }^{[19]}$. The immunohistochemical staining was carried out on $3 \mu \mathrm{m}$ thick paraffin sections using the streptavidin-biotin technique. After deparaffinization, sections were rehydrated in a descending graded ethanol series and washed twice with distilled water, five minutes each. Antigen retrieval was conducted for all sections using heat-induced epitope retrieval, through immersion of tissue sections in citrate buffer, $\mathrm{pH} 6.0$ at the autoclave $\left(105^{\circ} \mathrm{C}\right.$, for 20 minutes). The sections were incubated in $3 \% \mathrm{H}_{2} \mathrm{O}_{2}$ in absolute methanol for 30 minutes at $4{ }^{\circ} \mathrm{C}$ and followed by flushing with water. After treatment with blocking solution (Mouse stain kit, Histofine, Nichirei, Tokyo, Japan), the sections were incubated overnight at $4^{\circ} \mathrm{C}$ with the primary antibody for cTnT3 diluted in $0.01 \mathrm{M}$ phosphate buffered saline (PBS). Finally, the reaction was visualized with a 3, 3'-diaminobenzidine tetrahydrochloride $\mathrm{H} 2 \mathrm{O} 2$ solution for 3 minutes. Sections were washed in distilled water and finally counterstained slightly with Harris hematoxylin. For negative controls, incubation of sections was performed without the primary antibody ${ }^{[19]}$.

\section{Morphmetrical analysis}

To measure the cardiac cells' anti-cTnT3 staining affinity of, the optical densities of four different fields were evaluated at $400 \times$ magnification per a mouse $(n=6)$ using an image analysis software (Fiji image j; $1.51 \mathrm{n}$, NIH, USA).

\section{Statistical analysis}

The optical density values were analyzed with Independent Sample T-test using SPSS software program (version 16.0; Chicago, USA). The data were expressed as mean values $\pm \mathrm{SE}$ and $\mathrm{P}$-value $\leq 0.05$ was considered statistically significant.

\section{RESULTS}

Histological characteristics of the left ventricular wall

Examination of H\&E stained sections of the left ventricle: all the transverse oriented (Fig.1a) and the longitudinally oriented cardiac fibers (Fig.1b) appeared undistinguished. Homogenous staining of all cardiac fibers with undetectable difference among cardiac cells was noticed. In immunohistochemical staining, the negative control sections were carried out with the omission of the primary antiserum. A negative reaction in all cardiac cells was seen in these sections (Fig. 1c). While, the primary antibody (anti-cTnT3) immunostaining revealed two distinct staining affinities; a very low or no- and a high- positive affinity in the cytoplasm of cardiac fibers (Fig. 1d, e and f). In transverse oriented fibers, the cardiac cells exhibited low or no staining affinity were few, single and scattered among numerous high-positive affinity stained fibers. Their shape was polygonal or stellate (Fig.1 d). In the longitudinally oriented fibers, the highpositive affinity stained cells displayed transverse dark brown striations (Fig. 1 e and f).

\section{Histological characteristics of the inter-ventricular septum}

Examination of the H\&E stained sections did not declare any difference between cardiac fibers (Fig. 2a). In anticTnT3 immunostained-sections, the low or no-positive affinity cardiac fibers were grouped in the sub-endocardial regions (Fig. 2b). The statistical analysis of the optical density values for anti-cTnT3 staining affinities of cardiac cells revealed that optical density of the cardiac fibers with very low or no- positive affinity was significantly lower than cardiac fibers of a high-positive affinity $(2.5 \pm 0.01$; $3.04 \pm 0.19$, respectively; $P<0.022$ ). 

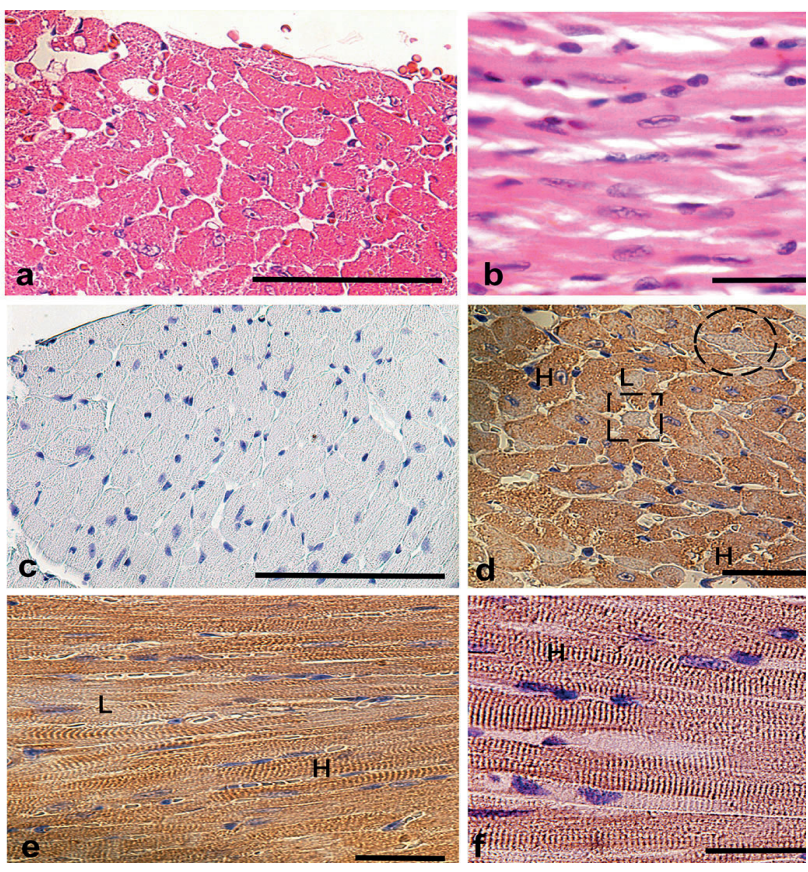

Fig. 1: Photomicrographs of sections in the left ventricular wall of an adult mouse heart. a: The transverse oriented cardiac cells showing a homogenous staining of all cells. b: The longitudinally oriented fibers showing a homogenous staining of all cardiac myofibers. c: A negative control stained section showing a negative cTnT3 expression in cardiac myofibers. d: A low or no- positive affinity of cTnT3 in single scattered cardiac fibers (L) in between high positive affinity immunostained myocytes $(\mathrm{H})$ is seen. Some of low immunopositive cells are polygonal (circle) and the others are stellate in shape (box). e: A low or no- positive affinity to anti-cTnT3 in single scattered cardiac fibers (L) in between the high positive affinity immunostained cardiac myocytes $(\mathrm{H})$ is noticed. f: The high positive affinity immunostained cardiac cells $(\mathrm{H})$ with brown striations are obviously seen. (a andb: H\&E, c: Harris Hematoxylin stain, d, e and f: Anti-cTnT3. The scale bars: $a, c$ and $d=200 \mu \mathrm{m} ; b=100$ $\mu \mathrm{m}$; e and $\mathrm{f}=50 \mu \mathrm{m})$.

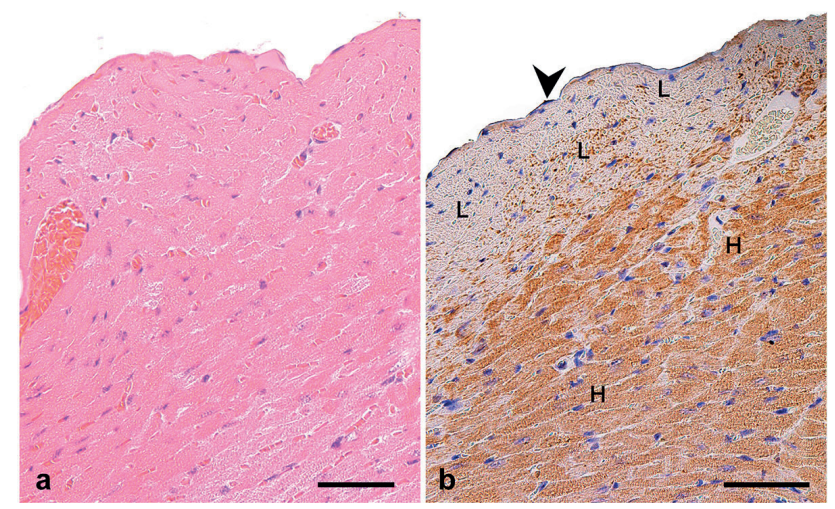

Fig. 2: Photomicrographs of sections in the inter-ventricular septum of an adult mouse heart a: All cardiac fibers showing a homogenous staining. b: The cardiac fibers expressed a low or no- positive affinity to anti- cTnT3 (L) in the subendocardial area (arrowhead) are gathered together. The high positive affinity to anti-cTnT3 $(\mathrm{H})$ is seen in the majority of cardiac fibers. (a: H\&E, b: Anti-cTnT3. The scale bar: $a$ and $b=200 \mu \mathrm{m}$ ).

\section{DISCUSSION}

In clinical practice, most of the previous cardiac investigations focused on non-invasive techniques such as Contrast Enhanced Micro-Computed Tomography ${ }^{[10]}$, Magnetic resonance Imaging "MRI"[20] and transmission electron microscope $\mathrm{e}^{[21]}$ to discriminate the different cardiac diseases. However, their high costs and inability to diagnose reasons for mortality are clear disadvantages of these tools. The immunohistochemical staining with antibodies were usually directed against specific ion channels localized to the membrane of conducting myocytes at high concentration ${ }^{[9]}$. Even in immunohistochemical labeling of cTn-I of engineered myocardial tissues, Xing et al. detected the cardiomyocyte-like tissues within the implanted scaffolds but could not discriminate between different types of cardiac cells ${ }^{[21]}$.

The formation of a troponin-tropomyosin complex in the cardiac sarcomere and the parallelization of the sarcomeric apparatus with the long axis of the muscular contractile fibers, as well as the fact that the cells of conducting system are devoid of sarcomeric formation directed use anti-Troponin-T3 immunostaining as a distinguishing parameter between the contractile and conducting fibers. In addition, Troponin- $T$ has been extensively investigated and found to be a sensitive marker for the detection of myocardial necrosis ${ }^{[16]}$.

Histological examination of the left ventricular wall and interventricular septum were chosen because the Purkinje network in the left ventricle is more extensive than that in the right ventricle ${ }^{[10]}$. The immunohistochemical staining with a commercially available anti-cTnT3 antibody showed obvious microscopic differences in the staining affinity between contractile and non-contractile fibers.

In this work, undetectable differences and homogeneously appearance of cardiac fibers in the H\&E stained sections disagreed with Ono et al. They could recognize the conducting fibers by H\&E stain via their light cell bodies that contain one or two nuclei and peripherally located few myofibrils ${ }^{[22]}$.

The immunohistochemical staining with the anti-cTnT3 antibody of mice hearts in the current study declared two affinities confirmed by densitometric analysis. The cardiac fibers of very low or no- positive affinity were significantly lower than cardiac fibers of a high- positive affinity. In transverse oriented fibers, the low densito-stained cardiac cells were polygonal or stellate in shape similar data had been described by Ono et al. ${ }^{[22]}$. In agreement with Yu et al., most cardiac cells showed highly positive immunostaining affinity in the form of transverse dark brown striations, especially in longitudinally oriented fibers ${ }^{[23]}$.

In this research, some penetrating cardiac fibers were distinguishable from the surrounding structures (contractile 
myocardium) with undetectable level of positive expression of cTnT3. A cTnT3-low staining affinity was reported in myocardial infarction or non-contractile cardiac cells such as conducting fibers ${ }^{[17]}$.

The number and location of these low or no-staining affinity cardiac fibers were different. They were either single scattered especially in the left ventricular wall or grouped in the subendocardial regions of the interventricular septum, these data were consistent with Stephenson et al. who localized the left ventricular Purkinje network of the rat in the apical region of the interventricular septum. They noted Purkinje fibers creating a scoop-like appearance, with the majority of the branches running on the endocardial surface towards the base ${ }^{[10]}$. In line, Ono et al. demonstrated the conducting fibers in the subendocardial region of both ventricles in the rat. The fibers were smaller in size when compared with myocytes and arranged in paralle ${ }^{[22]}$. These findings coincided with the fact that rats and mice hearts can beat 700 beats per minute ${ }^{[24]}$. In addition, Contactin-2, a cell adhesion molecule that is highly enriched transcript in conducting fibers, was restricted to a subendocardial network of cardiac cell in the murine ventricle ${ }^{[25]}$.

Despite reporting that free running Purkinje fibers are difficult to trace in any destructive sectioning technique because the fibers are lost following sectioning, immunohistochemical staining recognized the conducting fibers in many studies ${ }^{[10]}$. Anti-cTnT3 immunohistochemical staining of cardiac biopsies can assist in recognition of myocardial necrosis at autopsy, in cases of patients suspected to die from acute myocardial ischemia or due to heart block.

\section{CONCLUSION}

Anti-cTnT3 immunostaining could be added to the routine staining of cardiac biopsies. Further investigations are required to examine the cTnT3 in different cardiac disease conditions.

\section{CONFLICT OF INTEREST}

There are no conflicts of interest.

\section{REFERENCES}

1. Leone, O., Veinot, J.P., Angelini, A., Baandrup, U.T., Basso, C., Berry, G., et al. 2011 Consensus statement on endomyocardial biopsy from the Association for European Cardiovascular Pathology and the Society for Cardiovascular Pathology. Cardiovascular Pathology. 2012, 21, 245-74.

2. Reitz, B.A. 50th Anniversary Landmark Commentary on Caves PK, Stinson EB,
Billingham M, Shumway NE. Percutaneous transvenous endomyocardial biopsy in human heart recipients: experience with a new technique. Ann Thorac Surg 1973; 16: 325-36. The Annals of thoracic surgery. 2015, 99, 1875.

3. Liang, J.J., Hebl, V.B., DeSimone, C.V., Madhavan, M., Nanda, S., Kapa, S., et al. Electrogram guidance: a method to increase the precision and diagnostic yield of endomyocardial biopsy for suspected cardiac sarcoidosis and myocarditis. JACC: Heart Failure. 2014, 2, 466-73.

4. Au, H.T.H., Cheng, I., Chowdhury, M.F., Radisic, M. Interactive effects of surface topography and pulsatile electrical field stimulation on orientation and elongation of fibroblasts and cardiomyocytes. Biomaterials. 2007, 28, 4277-93.

5. Torrent-Guasp, F., Kocica, M.J., Corno, A.F., Komeda, M., Carreras-Costa, F., Flotats, A., et al. Towards new understanding of the heart structure and function. European journal of cardio-thoracic surgery. 2005, 27, 191-201.

6. Boyett, M.R. 'And the beat goes on'The cardiac conduction system: the wiring system of the heart. Experimental physiology. 2009, 94, 1035-49.

7. Boyett, M.R., Li, J., Inada, S., Dobrzynski, H., Schneider, J.E., Holden, A.V., et al. Imaging the heart: computer 3-dimensional anatomic models of the heart. Journal of electrocardiology. 2005, 38, 113-20.

8. Rentschler, S., Morley, G., Fishman, G. Molecular and functional maturation of the murine cardiac conduction system. In: Cold Spring Harbor symposia on quantitative biology, Cold Spring Harbor Laboratory Press, 2002, Vol. 67, pp. 353-62.

9. Yoo, S., Dobrzynski, H., Fedorov, V.V., Xu, S.-Z., Yamanushi, T.T., Jones, S.A., et al. Localization of $\mathrm{Na}+$ channel isoforms at the atrioventricular junction and atrioventricular node in the rat. Circulation. 2006, 114, 1360-71.

10. Stephenson, R.S., Boyett, M.R., Hart, G., Nikolaidou, T., Cai, X., Corno, A.F., et al. Contrast enhanced micro-computed tomography resolves the 3-dimensional morphology of the cardiac conduction system in mammalian hearts. PloS one. 2012, 7, e35299-e.

11. Corno, A.F., Kocica, M.J., Chappory, L.A., Moore, S.A. Inter-ventricular septum: New observations on the structure and function coupling. Basic 
Applied Myology. 2009, 19, 41-8.

12. Mesirca, P., Torrente, A.G., Mangoni, M.E. Functional role of voltage gated $\mathrm{Ca} 2+$ channels in heart automaticity. Frontiers in physiology. 2015, 6 .

13. Gordon, A., Homsher, E., Regnier, M. Regulation of contraction in striated muscle. Physiological reviews. 2000, 80, 853-924.

14. Venkatraman, G., Gomes, A.V., Kerrick, W.G.L., Potter, J.D. Characterization of troponin T dilated cardiomyopathy mutations in the fetal troponin isoform. Journal of Biological Chemistry. 2005, 280, 17584-92.

15. Fishbein, M.C., Wang, T., Matijasevic, M., Hong, L., Apple, F.S. Myocardial tissue troponins T and I: an immunohistochemical study in experimental models of myocardial ischemia. Cardiovascular Pathology. 2003, 12, 65-71.

16. Han, K., Flavin, R. Troponin $\mathrm{T}$ as a Diagnostic Marker in the Detection of Acute Myocardial Infarction at Autopsy. Int $\mathbf{J}$ Forensic Sci Pathol. 2014, 2, 28-9.

17. Berry, M.F., Engler, A.J., Woo, Y.J., Pirolli, T.J., Bish, L.T., Jayasankar, V., et al. Mesenchymal stem cell injection after myocardial infarction improves myocardial compliance. American Journal of Physiology-Heart and Circulatory Physiology. 2006, 290, H2196-H203.

18. Kumar, G.L., Kiernan, J. Special stains and H\&E. Connection. 2010, 14.

19. Jackson, P., Blythe, D. Immunohistochemical techniques. Teoksessa Bancroft, JD \& Gamble, M.(toim.) Theory and Practice of Histological Techniques. 2008, 6, 433-72.

20. Bordas, R., Gillow, K., Lou, Q., Efimov, I., Gavaghan, D., Kohl, P., et al. Rabbit-specific ventricular model of cardiac electrophysiological function including specialized conduction system. Progress in biophysics and molecular biology. 2011, 107, 90-100.

21. Xing, Y., Lv, A., Wang, L., Yan, X., Zhao, W., Cao, F. Engineered myocardial tissues constructed in vivo using cardiomyocyte-like cells derived from bone marrow mesenchymal stem cells in rats. Journal of biomedical science. 2012, 19, 6 .

22. Ono, N., Yamaguchi, T., Ishikawa, H., Arakawa, M., Takahashi, N., Saikawa, T., et al. Morphological varieties of the Purkinje fiber network in mammalian hearts, as revealed by light and electron microscopy. Archives of histology and cytology. 2009, 72, 139-49.

23. Yu, S., Zhu, Y., Li, F., Zhang, Y., Xia, C. Differentiation of human embryonic germ cells and transplantation in rats with acute myocardial infarction. Experimental and therapeutic medicine. 2014, 7, 615-20.

24. Noujaim, S.F., Lucca, E., Muñoz, V., Persaud, D., Berenfeld, O., Meijler, F.L., et al. From mouse to whale. Circulation. 2004, 110, 2802-8.

25. Pallante, B.A., Giovannone, S., Liu, F.-Y., Zhang, J., Liu, N., Kang, G., et al. Contactin-2 expression in the cardiac Purkinje fiber network. Circulation: Arrhythmia and Electrophysiology. 2010, CIRCEP. 109.928820. 




ترويونين تى 3 للقلب: معلم تمييز بين ألياف القلب الإنقباضية والناقله في ذكور الفئران البالغه

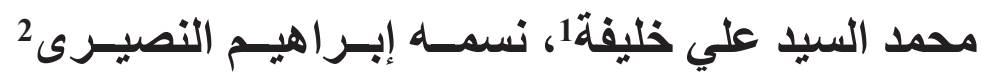

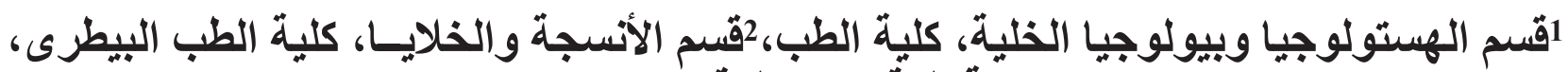

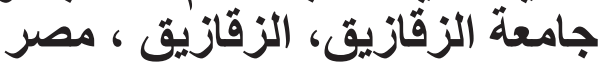

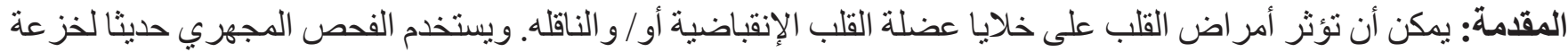

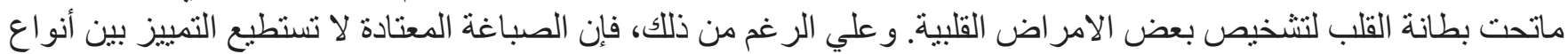
الألياف القلبية المختلفة.

الهـف من البحث: تهذف الدر اسة الحالية الي توضيح استخدام الصبغة المناعية المضادة للقلب" تروبونين تى"3 كأداة حديثة

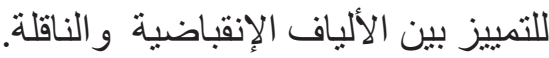



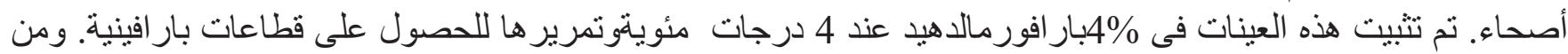

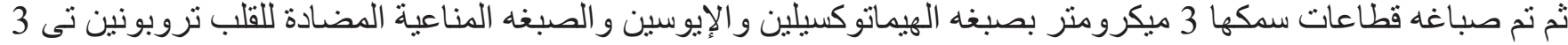

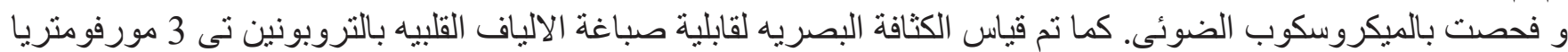
وتم تحليل القيم احصائيا.



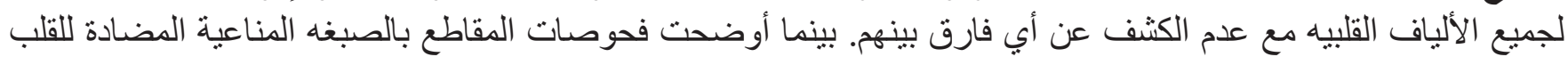

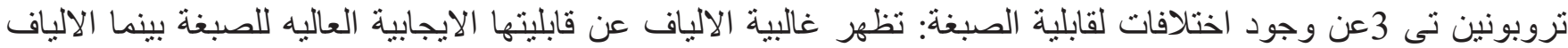

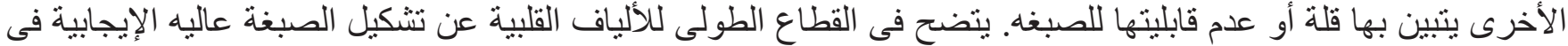

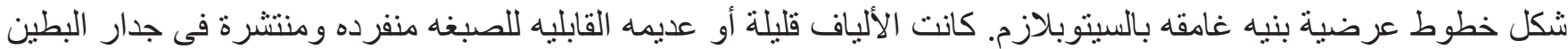

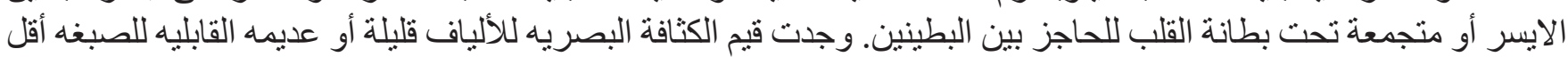

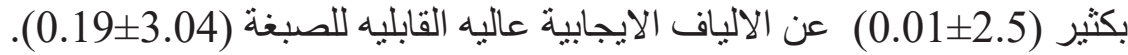
الإستنتاج : يمكن إضافة الصبغة المناعية المضادة للقلب" تروبونين تى "3 إلى الصباغات المعتاده في فحص خزعة القلب. 